\title{
Los bienes bibliográficos y documentales: un acercamiento a su problemática
}

\author{
María IDALIA GaRCía Aguilar \\ Centro Universitario de Investigaciones Bibliotecológicas \\ Universidad Nacional Autónoma de México \\ México, D.F.E-mail: pulga@ cuib.unam.mx
}

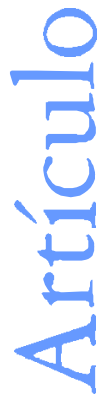

Trabajo recibido

el 28 de abril de 1999

Trabajo aceptado

el 26 de mayo

de 1999

\section{RESUMEN}

Este trabajo analiza el problema que representan los libros y documentos como elementos del patrimonio cultural. El tema aborda diversos enfoques sobre la vinculación que existe entre la sociedad y sus productos culturales en relación con la memoria colectiva. En la conservación del pasado y sus implicaciones, se señala aquí la ausencia de conceptos delimitados para ser aplicados en el trabajo de bibliotecarios y archivistas. Sin embargo, la problemática se complica dadas las connotaciones que subyacen a la concepción de estos tesoros del patrimonio cultural. El artículo presenta algunos conceptos con diferentes énfasis e ilustran la complejidad de la idea.

\section{THE BIBLIOGRAPHICAND DOCUMENTARY COLLECTION: AN APPROACH TOTHE PROBLEM \\ MARÍA IDALIA GARCÍA-AgUILAR}

\section{ABST RACT}

As products and indeed elements of cultural heritage, books and documents present librarians and archivists with diverse problems because of the lack conceptual parameters available to them as they carry out their work. These problems are analyzed in terms of the connection that exists between cultural products and society's collective memory and the implications of preserving the past. These problems are further complicated by the underlying conceptual connotations of these cultural treasures. The complexity of the problem is illustrated through the presentation of various concepts with different emphases.

\section{INTRODUCCIÓN}

I os bienes bibliográficos y documentales son algo más que objetos de gran valor — histórico y artístico. En el entorno del patrimonio cultural representan la aportación singular al conocimiento universal que cada colectivo realiza al conjunto de su cultura. D e ahí que sólo puedan ser explicados y especialmente comprendidos, desde el marco de las relaciones sociales e históricas que los han producido. Sin embargo , todo acercamiento tanto a sus características como a sus problemáticas, no puede desligarse de los tres órdenes que los sustentan: el jurídico, el institucional y el 


\section{Investigacón Biblideedógia v. 13 No. 26 enero/ junio de 1999}

cultural. Éstos condicionan por una parte las cualidades que los distinguen y por otra, determinan el conocimiento social de tan valiosos objetos.

Estos bienes presentan una problemática especial para todo estudio que pretenda el análisis e interpretación de su contenido; problemática que se refiere específicamente a su definición. Ciertamente esta dificultad no puede salvarse únicamente adaptando términos ya creados a los nuevos entornos de discusión. Esto no es posible en la medida en que el conjunto de bienes al que hacemos mención está recargado de un contenido simbólico y significativo que va más allá de su fuerte potencial como transmisores de cultura. Nos referimos a su condición y naturaleza patrimonial, a partir de la cual entendemos que su conformación y reconocimiento no es una tarea pasada sino permanente que consolida la memoria de un colectivo.

Por otra parte estos bienes de naturaleza cultural apuntalan la puesta en valor de otros objetos de igual envergadura y que definen en su totalidad las características culturales de una sociedad determinada. Sin embargo, ni el reconocimiento de su valor, ni la larga trayectoria del pensamiento conservacionista han conseguido afianzar la defensa de estos materiales en la consecuente lógica de su creación y como parte de un eslabón continuo e históricamente coherente. D e esta manera se ha permitido que la valoración de estos objetos se realice siempre con base en la necesidad de apuntalar un valor colectivo por sobre otro, lo que produce necesariamente una pérdida patrimonial.

Esta valoración no es gratuita ni ociosa, ya que depende del interés del Estado en rescatar sólo esa parte del pasado que es capaz de aglutinar los sentimientos colectivos de identidad, e igualmente de la evolución que haga el conjunto de lo social de la valoración de elementos culturales. Ambos procesos presentan ala cultura como un conjunto homogéneo que se deslinda de la sociedad, ya que ésta es completamente heterogénea y responde a la realidad cotidiana en constante movimiento. Una realidad en donde van a confrontarse los intereses sociales en busca de mejoras de sus condiciones pactadas, en el orden de lo social, económico, político y cultural.

Este espacio de pactos sociales que conocemos como Estado de derecho, implica el reconocimiento universal sin exclusividad y con equidad, en un solo espacio territorial de los derechos ciudadanos de cada uno de los individuos que lo componen. Pero la creación de este marco y sus particularidades nacionales son el resultado de la cultura como "el fenómeno a través del cual se formulan y jerarquizan los intereses y las necesarias transformaciones que requiere la renovación económica social."1 D e este modo la cultura y por tanto sus productos responden a una forma de vida determinada por la propia evolución de un colectivo. Sin embargo el derecho a la cultura y con ello la garantía de su permanencia y recreación, son reconocidos como derechos de segunda generación con el Pacto Internacional de D erechos Económicos, Sociales y Culturales de 1966 de la ONU. Con base en este marco, los Estados

1 Lian Karp. “Cultura y nación. Para el desarrollo de una conciencia crítica”. En Ingeriosparad Estur dodela Cultura México : UNAM. CRIM, 1992. p. 47 
asumen la tutela de los bienes culturales en beneficio del interés general por sobre el particular creando instituciones especiales e implementando políticas con este objetivo.

Ciertamente es imposible conservar la totalidad de los bienes culturales, del conjunto general siempre se seleccionan aquellos que por sus características son especialmente representativos de una cultura nacional. Pero la selección de los bienes a tutelar está influida por la ideología que abandera el grupo social dominante, la cual no necesariamente responde a la manifestación cultural de cada uno de los grupos sociales que componen al Estado nacional.

En efecto, al potencializar y distinguir sólo a una categoría de bienes en función de un interés determinado, una parte importante de la memoria histórica queda relegada sin que por ello sea desconocida, ya que la permanencia de esta memoria no depende de las estructuras sociales encargadas de mantenerla, sino de la representación y recreación de sus valores que hace un conjunto de la sociedad. Lo que explica el mantenimiento de formas diferentes de entender el pasado que subsisten en la actualidad, incluso de aquellas que por razones de construcción y evolución de un orden social han sido negadas como parte integrante de los rasgos distintivos de una cultura.

Pero incluso en aquel conjunto de bienes culturales queel Estado reconoce como valores potenciales de sustento cultural, existen objetos que por diferentes razones históricas han pasado a formar parte de un grupo diferente (lo que no significa excluyente) de bienes culturales que dada esta condición se encuentran permanentemente en riesgo de pérdida patrimonial y cuyo acercamiento a lo social se establece por otros senderos. Este es el caso concreto de los bienes bibliográficos y documentales, los que debido a su naturalezay características requieren necesariamentede un intermediario para poder ser comprendidos por el conjunto de la sociedad.

Este intermediario por norma general, es un investigador o profesionista con conocimientos especializados para interpretar el mensaje contenido en estos materiales y para contextualizar el objeto en cuestión, dentro de un período histórico determinado. Solo mediante este conocimiento, el objeto puede serpuesto en valor. Los conservacionistas entienden por este concepto, el rescate e interpretación de todos los factores culturales, sociales, económicos y políticos que posibilitaron la creación del objeto y explican por tanto su naturaleza cultural .

Ya que es necesaria la definición de los objetos a tutelar y el conocimiento de los valores quelos distinguen, para que una política de protección sea eficaz. Esta serála premisa básica del pensamiento conservacionista: no se puede proteger aquello que no se conoce. Como resultado de esto, el objeto cultural debe ser adscrito a una categoría general que incluya las características por las cuales es meritorio de protección y que a su vez comparte con otros objetos de la misma naturaleza.

En el acercamiento a los bienes bibliográficos y documentales dentro del esquema del patrimonio Cultural, se observa esta ausencia de conceptos generales básicamente determinada por la dispersión terminológica y conceptual que presentan 


\section{2}

como peculiaridad compartida la disciplina bibliotecológica y archivística. A simismo en el desarrollo disciplinar no se observa una clara tendencia que recupere este espacio de investigación y por tanto profundice en las problemáticas que se derivan de ella.

Esta situación permite la ausencia definida de estos materiales en las leyes encargadas de definir el alcance de la protección, ya que se requiere de un marco adecuado para que existan las condiciones que permitan, por un lado, el conocimiento, conservación y difusión de los bienes de naturaleza cultural, y por el otro, la continuidad de las políticas emprendidas para garantizar su adecuada permanencia en la posteridad. Este marco idóneo lo constituye el ordenamiento jurídico que si bien no es una panacea, representa un punto de partida imprescindible para defender el patrimonio cultural y a sus elementos de cualquier contingencia no previsible en el tiempo.

D e esta manera, la conservación de los bienes de naturaleza cultural requiere de dos frentes coadyuvantes: un marco jurídico que defina los objetos y el alcance de la tutela, y una política cultural que permita la implementación de programas específicos en concordancia con la obligación y la responsabilidad que se deriva de la aplicación de la ley. Pero ninguno de estos frentes puedeconsolidarse sin el concierto de la categoría jurídica que será la representación del bien a tutelar.

\section{LA NATURALEZA DE LOS BIENES BIBLIOGRÁ FICOS Y DOCUMENTALES}

Para que el reconocimiento de libros y documentos como elementos importantes de la construcción de la memoria colectiva logre solidez como discurso disciplinario, se necesita comprenderlos como productos culturales que responden a procesos históricos y sociales determinados. Asimismo hace falta que el alcance de la protección no se destine únicamente a la custodia de los testimonios del pasado, sino que garantice la continuidad en la conformación de los acervos bibliográficos y documentales en tanto que aportaciones al conocimiento, los primeros, y manifestaciones del acontecer social e institucional del colectivo, los segundos.

Sin embargo dadas las características propias de su contenido, es difícil hablar de una única definición universal. Ésta, no puede ser completa sin hablar del sujeto creador: el hombre. Sólo a partir de la identificación de un objeto como integrante dentro de una totalidad en la concepción de la realidad del sujeto, se puede precisar la idea de este Patrimonio. Contrariamente a lo que se piensa, es necesario ir construyendo de lo particularalo general y no viceversa, ya que son precisamente las cualidades de los sujetos en su relación social las que construyen elementos distintivos y diversos que los diferencian de otros. La construcción de este universo de significados mediante los que un colectivo se explica a sí mismo es un proceso histórico continuo que sealimentainterna y externamente de la recreación de esos símbolos heredados de generaciones anteriores. Empero la valoración de estos elementos 
culturales no discurre por este mismo proceso, y por el contrario significa la selección de unos por encima de otros en función de un interés determinado.

No obstante debemos partir de la naturaleza de los bienes objeto de la reflexión en tanto patrimoniales. Es decir, reconocemos en estos objetos una herencia de un pasado mediato e inmediato, un pasado que se corresponde con nuestro devenir histórico y en el que son estos objetos elementos de apuntalamiento. En estacaracterización es fundamental definir la tipología de los objetos culturales antes de posibilitar la creación o adecuación de marcos jurídicos que justifiquen la actuación política o instrumental sobre los objetos. Pero para ello debemos reconocer en primera instancia que estos objetos son físicos, es decir materiales transformados por la obra y la creatividad del hombre. Lo queno significa que existan elementos culturales que no necesariamente cumplan con esta característica, a los que denominaremos bienesintangibles y que en gran medida sirven parafundamentar el valor simbólico que adquieren aquellos que son de naturaleza física.

A éstos en tanto que objetos, desde la perspectiva del D erecho, se les reconoce la posibilidad de propiedad. Es decir son objetos de dominio, entendido éste como el poder que tiene un individuo para usar y disponer de lo que es suyo. A hora bien para el asunto que nos interesa, es necesario observar que de la sociedad dimana un E stado, y que éste cumple funciones políticas, administrativas y jurídicas. Estas últimas especialmente determinan el funcionamiento de la sociedad, ya que dela elección de ésta dependen y se especifican las características del Estado. Siempre que en éste el modelo aceptado sea la democracia liberal, en el cual la mayoría es representativa. ${ }^{2}$ Siendo así, al bien u objeto cultural dentro del ordenamiento jurídico sele distinguen dos peculiaridades: la primera es el tipo de propiedad del que es objeto y la segunda es la naturaleza a la que pertenece.

No obstante esta clara diferencia existente entre propiedad privada y pública, para efectos de bienes de naturaleza cultural el tipo de propiedad es secundario frenteal beneficio de la colectividad. D e esta manera, independientemente de la adscripción del dominio del objeto, se le reconocerála naturaleza cultural siemprey cuando sus particularidades cumplan con la definición que se establece para tal reconocimiento. He aquí la importancia del concepto genérico.

Si bien paralanoción de Patrimonio Cultural la puesta en valor del objeto será determinante para su introducción en un conjunto general, el problema de inscribir una cosa determinada en esta idea produjo múltiples discusiones en los foros de especialistas. Esta controversia encontró un equilibrio con la elaboración del concepto bienaltural por la Comisión Franceschini del parlamento italiano en 1964. Se trata de una idea aglutinadora que permite un amplio espectro en la identificación de los objetos culturales. Esta definición establece que un material (cosa) está dotado de

2 Crr. Jürgen Habermas. Tresmoddos dedammaacia: sdbed coneptodeuna pdítica ddiberativa. Valencia : Universitat de Valencia. Centro de Semiótica y Teoría del Espectáculo, 1994. 
un valor de civilización de forma testimonia,y en función de esto representa un elemento del patrimonio cultural. ${ }^{3}$ Esta definición constituyeel eje denuestro análisis.

La ventaja de esta categoríajurídica, la de la Comisión Fraceschini, no es más que aquélla que permite eliminar las condiciones subjetivas que implicaban la valoración estética e histórica predominante en los primeros tiempos del pensamiento conservacionista, y que permitía que elementos culturales de gran importancia quedasen relegados de este conjunto. Sin embargo dado el alto potencial de su instrumentación política, estos bienes siguen estando sujetos a diversas interpretaciones, las que efectivamentelibran mediante su inclusión en un ordenamiento jurídico ex profesa

El punto de partida principal parala elaboración de categorías de objetos culturales retoma nuevamenteal D erecho como inspiración y en función del dominio. Estos dos universos serán los bienes muebles y los bienes inmuebles, cuya diferencia principal radica en una cuestión de movilidad: los primeros la tienen y los segundos carecen de ella, pero ambos serán siempre sujeto de dominio.

En este tipo de propiedad no hay mayor conjetura, la cosa que pertenece a un miembro de la colectividad sea por adquisición o herencia, o de cualquier otraforma siempre será deél y podrá disponer de ella como mejor le parezca. Porque el ordenamiento jurídico de un Estado se presenta "como un todbunitarioque configura la sociedad como un entramado de derechos, deberes, potestades, garantías y fines en cuya urdidumbre los unos valen tanto como los otros, suponiendo su existencia 0 reconocimiento un freno límiteal reconocimiento que se efectúa [...] Lo que imposibilita atribuir a ninguno un valorabsduto por cuanto (cualquiera que sea el predominio) estará limitado por el reconocimiento otorgado a los demás [...]." 4 D e esta forma las leyes de los Estados modernos, ${ }^{5}$ reconocen que la propiedad de los objetos siempre será en dos sentidos: de beneficio y disfrute público, o en su aplicación de corte privado.

Esta es la característica principal que deberemos distinguir en todo objeto al que le reconozcamos su naturaleza cultural, la condición de interés social por encima de su condición de propiedad, siempre que el contenido a valorar del objeto sea representativo de la aportación cultural de los grupos sociales que integran un Estado.

Cuando hablamos de la propiedad de un bien, sea pública o privada, hacemos referencia a la tutelaridad, es decir la responsabilidad de hecho y los derechos sobre el bien en cuestión. En el caso de los bienes que recaen sobre latitularidad pública todos

3 Juan Manuel Alegre Avila. Evdudónyrégimenjuńdicoda PatrimmioHistónca Madrid : Ministerio de Cultura, 1994. p. 645

4 O scar Gómez Navas. “La protección del patrimonio histórico”. En CuadamosdeDœumentadóneIn fomaaón No. 62. p. 16. Las cursivas en el original.

5 Siempre que exista un estado de derecho, es decir un sistema que permita y asegure la existencia de los derechos fundamentales de los individuos. Lo que no significa que todo Estado por aplicación presente esta prerrogativa, aún cuando se supone que por su naturaleza los Estados Nacionales deben cumplirla en el propio ejercicio de su soberanía. Podríamos enumerar múltiples casos en que se dala condición deun Estado Nacional queno cumple necesariamente con el estado de derecho. 
comparten ciertas características: son inalienables, inembargables e imprescriptibles. ${ }^{6}$ Por lo primera entendemos que no pueden ser enajenados; es decir que su dominio o derecho no puede ser traspasado a otro titular. Por lo segundo entendemos que no pueden ser sujetos de embargo, lo que significa que no pueden ser retenidos por ninguna persona jurídica o moral en razón de un ordenamiento judicial. Y por lo tercero, entendemos que no prescriben, es decir no pueden cambiar de dominio ni por razón del tiempo dadas las condiciones previstas en las leyes. Estas condiciones no se cumplirán con los bienes considerados de titularidad privada pues pertenecen a otro régimen.

Por otra parte la noción genérica de la naturaleza cultural presenta dificultades, porque analizada desde cualquier punto de vista o intención debe considerar los aspectos culturales e históricos, y por tanto tomar conciencia de que nos acercamos a una problemática de estudio queno estáfija en el tiempo y por medio de la cual se establecen lazos estrechos entre generaciones de individuos. Partimos de la idea de que cada grupo humano se reconoce en ciertos valores heredados por sus antecesores, y que tales valores constituyen elementos patrimoniales que identifican a sus integrantes. Finalmente una cultura responde a un saber acumulado por una sociedad determinada, que rescata y reconoce los valores del pasado de forma diferente.

Esta valoración siempre le es añadida al objeto, recargando o recreando un significado simbólico determinado por encima de la particularidad física del objeto mismo (forma, color, materia, etc.). Ciertamente en esta valoración es el valor histórico lo que predomina, ya que estevalor histórico, reconoce todo lo que ha existido alguna vez y forma un eslabón de una cadena continua siempre condicionada por lo anterior?. Pero la amplitud de este valor que permitiría la conservación absoluta de los bienes culturales, obliga a acotar con base en la noción de testimonio, lo que significa su capacidad de representar un período distintivo en el devenir histórico delo social.

En la elaboración de conceptos genéricos, el concepto testimonio no constituye únicamente una cuestión de contenido; es decir no representa una serie de objetos que conforman un inventario de obras individuales o colectivas, las cuales se han elaborado alo largo de una historia particular para una sociedad. No obstante ésta es la tendencia general que se presenta en la elaboración de categorías para estos objetos. La problemática deviene más de forma, es decir de definición.

Este será por tanto el punto nodal de partida para elaborar la categoría de los bienes bibliográficos y documentales en su dimensión patrimonial. En efecto, por su naturalezafísica, tal como lo hemos mencionado, tales bienes pertenecerán ala categoría genérica de los bienes muebles en tanto que pueden físicamente ser transportados sin que ello desacredite su valor cultural.

Pero el reconocimiento de lo objetos debe considerar en primera instancia la función social que cumplen, ya que sin ésto la referencia en tiempo y espacio sobre el

6 Diciananiojuńdico Espasa Madrid : Fundación Tomás Moro : Espasa Calpe, 1993. p. 115

7 Aloïs Riegl. El altomodemoalosdbamentos Madrid : Visor, 1987. p. 24 
curso de las acciones humanas perdería anclaje. Estos bienes son comúnmente el libro y el documento, en ambos, se manifiestan giros radicales en la historia de la humanidad; la creación de algo más importante aún que el lenguaje hablado, una herramienta trascendente y revolucionaria: la escritura. Ésta marca, sin lugar a dudas, un punto capital en la historia del pensamiento y en los modos de comunicación. Con este instrumento la memoria del hombre se vuelve física y permanece. Pero al igual que la escritura sirvió para transmitir los acontecimientos de la historia humana, también pasó a formar parte del proceso creativo del pensamiento humano.

Porque el complejo proceso de elaborar signos y la capacidad de combinarlos para hacer un discurso, es sin lugar a dudas laverdadera transformación que diferencia al animal de la naturaleza del homosapiens A partir de entonces todo cambia, el hombre ha creado algo que no será capaz de controlar del todo, algo que ya no le permitirá olvidar.

Se dice que con la escritura la historia del hombre comienza formalmente, que antes de ella sólo había tinieblas y vaguedad en la memoria. Para que ésta fuera posible se invirtieron siglos en su desarrollo, hubo que conceptualizar y darle forma a los sonidos de la lengua, proceso no fácil y menos aún provocar con todo ésto la transformación del orden social. Fue necesario el cambio de la cultura oral a la cultura escrita: "Para conservar lo didofue menester inventar la escritura." 8

Con el establecimiento de las sociedades, de las jerarquías y primordialmente de las instituciones, aunadas a la herramienta de la escritura, el acontecer cotidiano, es decir la relación directa entre los seres humanos, produjo el documento. Este objeto funcionó y sigue desempeñando el papel de testimonio fidedigno de lo acontecido; con él no hay acontecimiento humano que no deje una traza en el camino, aunque este sendero pueda volverse laberinto. El documento es testigo y juez de la historia, en él se encuentra lo que fue eincluso lo que no pudo ser. Es el testigo silencioso einmortal de la obra humana, de todos y cada uno de los detalles, de las particularidades, de las singularidades de la vida social del hombre. Pese a su importancia, a prueba de toda duda, un documento aislado y solitario en el tiempo es un secreto guardado e impenetrable que deja sólo ver un poco; pero en conjunto el grupo de documentos permite reconstruir un pasado no necesariamente anterior, sino que incluso puede estar completamente alejado del momento en que son analizados los documentos.

En principio documento y libro no habían delimitado sus fronteras y el primero servíaúnicamente como elemento probatorio de lo hablado; el segundo en cambio, abrió el universo al pensamiento del hombre. Pero ninguno de los dos tiene absoluto sentido sin la escritura, esta representa la transición del mundo oral a un mundo diferente dondeya no sólo se habla sino donde se fijan los pensamientos para la posteridad. ${ }^{9}$ Pero ese documento originario se volvió rápidamente un lugar seguro

8 Julián Marías. "El libro en el pensamiento y la continuidad histórica”. EnLaalturaddlibro/ coord. Fernando Lázaro Carrater. Madrid : Fundación G ermán Sánchez Ruipérez : Pirámide:1988. p. 29

9 Henri-Jean Martin. Histaree paxair del'enit. París : Librairie Académique Perrin, 1988. p. 96 
de referencia sobre el pasado; ese paso inseguro se afirmó, y la herencia de las generaciones humanas se entregó ya no únicamente a las más cercanas, sino que las lejanas en el tiempo y en la geografía se acercaron irremediablemente.

La característicaintrínseca de la escritura acercaal hombre a la conciencia socialy a esa verdad de los hechos, algo que no siempre beneficia a los poderes imperantes de la sociedad. Por ello al principio no fue un beneficio del ciudadano común sino privilegio de los grupos go bernantes. En diversas civilizaciones el uso de la palabra, tanto oral como escrita, estaba destinado únicamente a los sacerdotes, los guardianes de un saber 0 a los go bernantes depositarios y herederos de un pasado. No son raros los casos en la historia de la humanidad en los que no se desea dejar testimonio escrito de lo acontecido. Incluso no todo el pensamiento de los grandes hombres de la antigüedad hallegado hasta nuestros tiempos por su propialabor, sino por la tarea de otros (Platón hace hablar a Sócrates en sus diálogos).

No se puede negar que el origen del documento es el de ser testimonio, sin embargo ese lugar que le otorga su función social, también deja oculto su verdadero valor cultural. El hecho no es gratuito, el siglo XIX que vio nacerla ciencia histórica y el interés por conocer y preservar los acontecimientos del pasado es también el que le proporciona su cédula de identidad al documento. El asunto es un poco complejo. Cuando la Historia se establece como ciencia docta y se crean las Acadkmiastan ligadas a un sentido de identidad nacional, el documento sirve para "probar" que un hecho aconteció, que lo que ahí se narra, existió. Es un objeto de la cultura que proporciona fechas, nombres y lugares, que en buena medida denuncia los detalles de la historia de los hombres.

No sucedió así con el libro, que se acercó a la sensibilidad humana dándole cuerpo a ese cúmulo de experiencias, recreadas y vivenciadas (aunque no exclusivamente) por la imaginación. El libro sintetiza la memoria y la experiencia de su autor, interpreta el mundo "vivo" de las sociedades. No enlaza únicamente con el pasado, sino que logra conjugar los tres momentos del tiempo: pasado, presente y futuro, que se entremezclan y nos muestran la complejidad del espíritu humano.

A ninguno de estos objetos se le puede negar su valor cultural, en tanto que son producidos por el cúmulo de conocimientos, experiencias y la sensibilidad de una sociedad. Son, en suma, el resultado de la forma en que cada una de las culturas antiguas elabora su escritura, establece su relación con el pasado, y recrea su propia historia. La elaboración de testimonios escritos varía de una cultura a otra no sólo en lo que se refiere al material sobre el que se asientan los símbolos y que responde necesariamente al entorno en que se desarrolla esa cultura, sino también en la forma misma de la escritura: pictográfica, ideográfica, cuneiforme, etc. Varían los tiempos y en las formas de los signos.

Tanto la historia del libro como la del documento se encuentran ligadas irremediablemente a la historia de los soportes y de las diversas culturas, hablamos de la historia misma de la humanidad. Pero quizás nadie imaginó que aquellos "signos", y más aun el uso de ellos, pudiera convertirse en un universo inimaginable de posibilidades 


\section{8}

para el ser humano y su entorno. Tanto el documento como el libro se transformaron en necesidades sociales. Por ello no es extraño que el momento en el que el libro florece y se fortalece como una necesidad, aparezca la imprenta, justo en el momento histórico en que se requerían más libros. ${ }^{10}$

Pero en ese mismo momento se establecen los roles diferentes que ambos objetos van a jugar en la historia: el libro se difunde y alcanza más terreno porque su recién adquirida naturaleza así lo permite; se expande y se dice que difunde la cultura. Mientras que el documento pudiendo gozar de los mismos favores no puede ser difundido con el mismo entusiasmo, pues no ha sido creado con esa intención. Todo lo contrario, el documento no es público y ni siquiera es social, desempeña un papel más delimitado en la sociedad. Mientras el libro es público y social, el documento es particulary único, el primero puede ser de conocimiento común, pues la información en él contenida no requiere de un complejo saber para ser interpretada; en tanto que el documento no es un objeto que pueda ser entendido por el conjunto de la sociedad.

El documento requiere necesariamente de un mediador para ser interpretado, pues ha sido elaborado por una persona que cumple con un determinado papel social, para el desempeño del cual debe poseer un conocimiento particular. D e hecho entre más separado temporalmente se encuentre el documento de quien lo analiza, más se complica su comprensión.Porque su existencia se entrelaza con las vidas de los hombres en tanto que individuos sociales. El documento es testimonio, da fe del acontecimiento de forma similar a como lo haría un testigo ocular; su función social responde más a las instituciones de la sociedad y nos habla del funcionamiento de éstas. Si el libro logra por su naturaleza integrar una visión, el documento desmenuza esa misma visión permitiendo un acercamiento a la realidad que se asemeja más a la figura que surge en un caleidoscopio, que se modifica con cada nuevo movimiento.

El documento nace oculto, el libro nace como señor de la calle. Por eso en ocasiones éste es perseguido, en otras prohibido y en más aún controlado. Es no sólo una muestra más de la cultura humana sino el único objeto capaz de liberar al hombre de sí mismo. A través de él, es posible no sólo construir sino, más aún llegar a creer en un mundo diferente. El verdadero valor de este objeto cultural es hacer manifiesto el pensamiento del hombre y más aún transformarlo, lo cual implica riesgos para la libertad del espíritu humano, y es por eso que el contenido puede ser censurado. Sobran ejemplos históricos de libros quemados y autores perseguidos, del establecimiento de normas e instituciones destinadas exclusivamente a controlar en la mayor medida posible. Porque este objeto que se vuelve recipiente, condensa dentro de sí el propio desarrollo del pensamiento del escritor. E se contenido va enlazando de forma continua el razonamiento de los hombres, las ideas que van surgiendo en el tiempo e intenta responder a esas preguntas universales que aún angustian a la humanidad.

10 José O rtega y G asset. Misóndd biblideearia Málaga : Asociación Andaluza de Bibliotecarios, 1994. p. 22 
El pensamiento privado de un hombre se vuelve a través del libro, un asunto de dominio público, y no quedan ya secretos inconfesables ni pasados olvidados. Es ésta la diferencia sustancial entre el documento y el libro: la función que cumplen en el marco de las relaciones sociales. Para funcionar como testimonio, el documento requiere ser validado por el poder público o la acción social que lo genera. Nunca se elabora de forma gratuita sino que responde a una necesidad, aspecto que se explica por qué el documento sólo puede entenderse en relación con otros documentos. El libro por su parte no requiere de validación y conforma una unidad que perfectamente se explica a sí misma; el conjunto lo enriquece, pero no lo justifica.

El documento sirve para testimoniar el paso del tiempo y el curso de las acciones humanas; el libro transmite un mensaje que busca un interlocutor sin importar ni el lugar y mucho menos el tiempo. ${ }^{11}$ Pero esto ha sido así únicamente debido a la función social que le ha tocado cumplir a cada uno de éstos.

Esta función, dentro y fuera de la relación social de cada uno de estos objetos (libros y documentos) también ha permitido que nos duelan o que les prestémos mayor importancia a las pérdidas irreparables que han sufrido las bibliotecas pero no los archivos. Quizás la herida más profunda en la historia del pensamiento humano siga siendo la destrucción de la Biblioteca de Alejandría. Esa increíble colección de libros devorados por la vorágine del fuego. Nos duele porque cuando el libro se expande en el mundo cotidiano sevuelvealgo "nuestro"; hemos aprendido a valorarlo no solamente por su valor instrumental sino por el papel que hajugado en la historia. Cuando este tipo de acontecimientos ocurre la cultura lo resiente, porque el libro está revestido de todo un simbolismo cultural que se traza desde la invención de la escritura. Nos parece irreparable la pérdida de tales objetos porque representan la pérdida de un momento del pensamiento, sin el cuál lo que precede se queda considerablemente incompleto. Ni Copérnico, ni Newton, ni Kant, ni D escartes entre tantos otros podrían haber desarrollado conocimientos fundamentales para la cultura, de no haber tomado en cuenta la participación del los hombres del pasado. Por ello la ausencia de conocimientos siempre produce una sensación de vacío, algo se queda de alguna manera sin sentido. ${ }^{12}$

Si no ocurre de lo mismo con el documento es porque éste ha quedado en cierta forma marginado de estejuego social. Pero esta situación no es fortuita, todo lo contrario: la verdad oculta de un documento puede trastocar todo el orden colectivo, y por esta singularidad su función social está mayormente relegada. Esto se explica porque la naturaleza del documento está directamente vinculada al funcionamiento de la sociedad y especialmente de las instituciones que se encargan de regular las relaciones entre los individuos y los poderes otorgados al Estado.

11 Mercedes D exeus. "El libro como testimonio cultural”. En PatrimmioHistónca Santander : Universidad de Cantabria : Asamblea Regional de Cantabria, 1994. p. 34

12 Julián Marías. Op at. p. 61 


\section{Investigacón Biblideedógia v. 13 No. 26 enero/ junio de 1999}

Más allá de representar el equilibrio de los pactos sociales, el Estado también regula el ejercicio del poder, el que pese a ser dimanado técnicamente del ejercicio soberano del pueblo pierde accesibilidad para el ciudadano común en la práctica de la vida cotidiana. La estructura del Estado ha sido previamentelegitimada parafuncionar administrativamente de acuerdo con un margen que las propias leyes establecen. Pero cuando el poder trastoca el concierto social puede volverse siniestro; es entonces cuando el documento se convierte en garante de esos acuerdos ya reconocidos. Sin embargo recuérdese siempre que esta garantía debe ser universal y alcanzar al conjunto de los ciudadanos, y por tanto no debe confrontarse el derecho a la información, sobre todo frente a la vulneración de las garantías individuales. Este es el abismo que separa especialmente al documento y le da una dimensión patrimonial (testimonial y jurídica), que el libro ya no comparte.

\section{DE LA FUNCIÓN A LA DEFINICIÓN}

Q ueda claro que los libros y documentos son considerados como objetos de cultura, y que cada uno tiene funciones sociales determinadas que los caracterizan y diferencian. Empero definir con claridad, exactitud y precisión el significado de una palabra respecto de estos objetos es algo que presenta enormes dificultades. Esta definición evidentemente debe corresponderse con la naturaleza física del objeto que se percibe. En este sentido el uso de la palabra documento ha propiciado múltiples confusiones y no existe una definición unitaria y conceptual, especialmente en el terreno de lo social. Por lo que toca a la bibliotecología y la archivística, son precisamentelos especialistas quienes provocan esta confusión entre dos territorios diferentes y complementarios. Las reflexiones hasta ahora expuestas nos obligan a revisar las definiciones que predominan en estas disciplinas por lo que se refiere a libros y documentos. El propósito de esta revisión sería mostrar la imposibilidad de usar estas definiciones para apuntalar el pensamiento conservacionista que fundamenta la Teoría del patrimonio cultural. Observaremos a continuación que en algunas de éstas, el documento pierde completamente su identidad propia. Así, la American Library Association define al libro como:

Colección de hojas de papel, pergamino u otro material unidas entre sí de alguna manera, ya estén impresas, escritas o en blanco, e independientemente de un receptáculo o de una caja. Según laUNESCO, publicación literaria y no periódica que contiene cuarenta y nueve páginas o más. O bra literaria o una de sus partes más importantes;

$\mathrm{Y}$ al documento como:

Material de distintas clases en el que se registra un trabajo, parte de éste, o muchas obras. Entre los diversos tipos de documentos figuran los libros y el material similar a ellos, hojas impresas, gráficos, manuscritos, 
grabaciones sonoras y de vídeo, películas cinematográficas y ficheros de datos legibles por la máquina. En reprografía, un solo trozo de papel que contiene texto u otra materia gráfica en una cara o en ambas. Expresión abreviada de un documento o de una publicación oficial. ${ }^{13}$

Como se puede observar el documento puede ser todo, incluso un libro. Y si por éste entendemos una obra literaria de determinado formato, entonces ¿qué es el documento?. Esta es una de las contradicciones más difíciles de abordar, pues si el documento es un material de distintas clases, resulta que le estamos negando un sentido propio.

Para Buonocore,

[...] la idea exacta y cabal del libro debe abarcar sus dos aspectos: como substanciay como forma, como contenido y como continente, como espíritu y como materia, como concepto y como objeto físico, ya que la cobertura bajo la cual se presenta no es lo esencial sino lo accesorio [...] diríamos que libroesaalquier porión pequeñaogande, di pensamientohumano, trasmitida por ecrito o por los śmbolos deuna espeialidad, difundida por proedimientos meánices, fotomeánicos oaudiqparlantes y commicadal próïmousandb mateiales dearalquier daseyadqptandbaralquier foma oextensión [...] es siempre un mensaje del hombre, porque contiene en poco o en mucho ideas y emociones. Si falta este elemento vital y anímico, aunque tenga la forma física del libro, propiamente no es tal sino mero papel impreso.

Mientras que para definir documento, dice:

Esta palabra tiene distintos significados, según se le considere desde el punto devistajurídico, documentalistapropiamente dicho 0 archivológico [...] En unapalabra, documento es todo aquello que tiene una base material de fijeza y que puede servir como elemento de prueba o de enseñanza de un hecho o una cosa. [Así...] el concepto de documento tiene [...] una gran amplitud que lo hace casi sinónimo de fuente de conocimiento. ${ }^{14}$

En esta segunda definición el libro posee un valor "humano" de gran calibre, en tanto que el documento sólo tiene fuerza como elemento probatorio y testimonial, pero esta condición minimiza el contenido cultural del documento. Se mantiene de esta manera una indefinición no sólo material sino conceptual.

Para Martínez de Souza, el libro puede ser el:

13 GlosanioALA: debiblictedoǵayảeniasdelainformacón Madrid : D íaz de Santos, 1988. p. 39y 116

14 D omingo Buonocore. DicionariodeBiblictedoǵa $2^{a}$ ed aumentada. Argentina : Marimar, 1984. p. 281 y 173 
Conjunto de hojas de papel, papiro, pergamino, vitela u otra materia escriptórea, manuscritas o impresas, reunidas en el lomo por medio de cosido, encolado, anillado, etc., con cubierta de madera, cartón cartoncillo, pergamino, cartulina, papel, u otro material formando un volumen. / Publicación unitaria que consta de 49 o más páginas. / O braliteraria, técnica o científica de extensión suficiente para formar volumen. / Cada una de ciertas partes principales en que suele dividirse el contenido de una obra, y los códigosy leyes de gran extensión./ Registro donde se hacen constar ciertos datos o que sirve para llevar la contabilidad.

En tanto que el documento será la:

Expresión del pensamiento (biografía, relato, texto laudatorio, funerario, testamentario, etc.) por medio de signos gráficos (letras, dibujos, pintura, etc.) sobre un soporte (piedra, pergamino, papel, lienzo, película, cinta, etc.). / Información registrada que se considera una unidad en un proceso de documentación./ Diploma, carta, relación u otro escrito queilustra acerca de un hecho, principalmente histórico. / En diplomática, testimonio escrito sobre un hecho de naturaleza jurídica, redactado con arreglo a ciertas formalidades destinadas a conferirle autoridad, fe y fuerza probatoria. / En archivística, conjunto constituido por un soportey lainformación que contiene, utilizable con fines de consulta o como prueba. / Pieza de archivo o manuscrito. ${ }^{15}$

En esta tercera definición vuelve a presentarse la contradicción conceptual que afecta al documento. Se advierte que al acercase a este objeto con una finalidad, cada disciplina le otorga un significado diferente. Pero aunque no podemos afirmar con certeza qué es cada uno de estos objetos, lo que queda consolidado es la fuerza probatoria del documento en contraposición a la forma física del libro.

El Librarian's G lossary da la siguiente definición de libro, aunque la tendencia es aquí a referirse a un conjunto de definiciones:

A set of blank sheets of paper bound along one edge and enclosed within protective covers to form a volume, especially a written or printed literacy composition presented in this way. / A division of a literary work, which is separately published and has an independent physical existence, although its pagination may be continuos with other volumes. / At a UNESCO conference in 1964 a book was defined as a non-periodical printed publication of at least forty-nine pages, exclusive of cover pages. / A collection of manuscripts or printed leaves fastened together to form a volume or volumes, forming a bibliographical unit.

Altiempo queel documento tampoco se salvade poseer diversas definiciones:

15 José Martínez de Souza. Dicionariodebiblidoǵayäenaasafines $2^{2}$ ed aumentada y actualizada. Madrid : Pirámide : Fundación Germán Sánchez Ruipérez, 1993. p. 518 y 284 
A record-made on a more or less flat surface or on a surface admitting of being spread flat when required, made of paper or other material fit for easy handling, transport across space, and preservation through time- of thought created by mind and expressed in language or symbols or in any other mode, and/ or a record of natural or social phenomena made directly by an instrument without being passed through the human mind and woven into thought created and expressed by it. / Material of any kind, regardless of its physical form and characteristics, on which information has been recorded / A record, usually inscribed or written, which conveys information, and which is relied on to establish facts. It is now taken to include any form of graphic, acoustic or hatpin record (book, cutting, map, manuscript, drawing, periodical, etc.) . / A work recorded in language or symbols, or by other means. / Any form of recorded information, whether indited, alphanumeric, pictorial or auditory. The tendency has developed in the last few years when referring to the contents of a library or documentation center to consider all the individual items as documents on the grounds that various kinds or record are included in the collections. By D ocument is often meant any kind of record, whatever its data or form, and whether it is on paper, parchment or film or any other material. The result is that document is being when book or any other form of record is meant. ${ }^{16}$

La orientación sigue siendo la misma, el libro más o menos posee una forma material que lo distingue, mientras que el documento es multiforme y multiuso en la mejor acepción de los términos, y aunque se le reconozca su valor instrumental como medio de transferir significados en la línea del tiempo, sigue sin poseer una serie de características que lo hagan único eincomparable. Es precisamentela posibilidad como medio transmisor la que lo aleja de ser un continente físico definido, mientras que para el libro sí se presume una estrecha relación con el pensamiento humano.

En un diccionario técnico encontramos una referencia escueta y sencilla para definir libro:

A written or printed work of some length on consecutive sheets of paper fastened or bound together in a volume.

Sin embargo, la definición se complica para la palabra documento:

Writings, records of official publications of government and business. / Any material of a factual or informative nature, regardless of format. / Any material admissible as part of an archive. ${ }^{17}$

16 HanodsLibranian'sgossary. of temsusedinlibrarianship dbamentationandthebookscratts $5^{\mathrm{a}}$ ed revised. Great Britain : G ower, 1984. p. 90 y 246-247

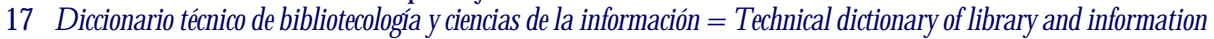
saene New Y ork : Garland Publishing, 1993. p. 34 y 91 
Aquí, el libro sigue manteniendo esa forma física ya definida, al tiempo que el documento en términos generales es totalmente indefinido. Parece quedar claro que el documento cobra sustancia sólo al acercarse 0 a una disciplina 0 a un tipo de institución. Esta cercanía que posibilita su distinción está basada directamente en su función social, que es una característica material propia.

0 tro diccionario más básico y no tan cercano al terreno delos especialistas define así al libro:

Avant d'être un ensemble de feuilles de papier primées et réunies en un volume broché et relié, livre en tant que support de l'ecriture revêtit des formes les plus variées, quón rencontre encore dans certaines cultures: tablette d'argile, pierre (en cèle ou en prisme par example), coquillage, volumen, en papyrus, en parchemin ou en papier, rouleau de pie, paravent, feuillets ou plaquettes de matériaux rivers, paravent, feuichillets ou plaquettes de matériaux divers (écaille de tortue, os, bois, bambou, jade, palmier, de galloche ou de bouleua, ivoire, etc.) On le définit actuellement comme un support maniare de l'écriture permettant la diffusion et la conservation d'un texte, definition qui neglige par croissante del'image dans le livre. Il est élément du patrimoine en tant que réalité intellectuelle et metérielle, et l'objet étude de la bibliologie.

Esta misma fuente define al documento como:

Ensemble d'un support d'information, des donnés enregistrées sur ce support et de leur signification, servant à la consultation, l'étude, la preuve, etc. Tout objet matérial - naturel ou artificiel- peut être utilisé comme document. Les collections des bibliothéques se limitent le plus souvent aux documents manuscrits, imprimés (bibliographiques ou iconographiques) et audiovisuels. ${ }^{18}$

Podemos deducir del conjunto de las definiciones aquí expuestas ${ }^{19}$ que sólo utilizamos para exponer las dificultades que encontramos desde el primer acercamiento a los bienes bibliográficos y documentales, que se concibe al libro como un objeto con características materiales específicas que le otorgan una personalidad concreta y delimitada. En tanto que el documento puede ser cualquier mensaje y aparecer bajo cualquier forma física. Estas particularidades conceptuales de ambos objetos impiden su introducción al conjunto del Patrimonio Cultural, pues desde esta perspectiva ninguno de esos objetos es fácilmente discernible. Ya que frente a todo esto, tenemos también que considerar que la puesta en valor de tales objetos presenta

18 SophieD anis. Peitdidionnairedel'appretivbiblidhéaireauledbamentdanstasseséats Villeurbanne: ENSB, 1985. p. 83 y 48

19 No se complica deducir por el uso común (incluidos los especialistas) de la palabra documento, que éste se convierte en un concepto que bien podríamos llamar "comodín", es decir la palabra tie ne tantas utilidades conforme se acerque a un determinado contexto. Esto hapropiciado que se re quiera hacer especificaciones y contextualizaciones cada vez que se le utiliza. 
riesgos importantes de interpretación. Especialmente si consideramos como universo paralelo de aplicación el papel e importancia de las instituciones encargadas de su conformación, conservación y difusión.

En este sentido las instituciones adquieren su propio valor cultural, que evidentemente va más allá de la sola concepción material y utilitaria que los especialistas le otorgan a los objetos en cuestión. D e esta manera y en este enfoque lo que se considera para su preservación no es la forma física que tienen, sino la función social que cumplen, y especialmente el contenido que poseen en relación con la cultura y sus manifestaciones.

En efecto, si se permite la existencia de categorías de protección y tutela con exclusiones importantes 0 , en su defecto, se antepone un valor preponderante por sobre otros, estaremos también poniendo en gran riesgo unos bienes que por sobre todo debemos respetar por una razón muy simple: no nos pertenecen, únicamente los cuidamos para asegurar su permanencia en el futuro, que es el lugar que les corresponde. Porque su naturaleza es siempre patrimonial, deben heredarse permanentemente.

Si por documento estamos entendiendo cualquier objeto, cuya única particularidad es que tiene un mensaje, y que éste puede (o más bien debe) transmitirlo, estamos perdiendo un referente objetivo que impedirá que un ordenamiento jurídico sea en este sentido específico. Si entendemos que documento es todo aquello que testimonia algo, la cosa se complica aún más. Servir de testimonio es algo que puede hacer cualquiera de los bienes muebles e inmuebles, categorías jurídicas que existen y componen el Patrimonio Cultural.

\section{MARCANDO EL HORIZONTE DE APLICACIÓN}

Tenemos la certeza de que el contenido de los libros presenta una continuidad histórica, valor que nunca podríamos negarle al documento. A este objeto menos que a ninguno, porque es la manifestación de dicha continuidad y gracias a ella es que nuestra vida como seres humanos en sociedad adquiere plenitud y sentido. $\mathrm{Si}$ bien es cierto que el libro nos proporciona una visión integral de una cultura determinada en un lugar y espacio específico; sin el documento el libro carecería de elementos para poder completar su función social. Podemos mencionar innumerables ejemplos: en los albores de la imprenta un libro podía ser público en tanto cumpliera con las normas sociales establecidas para ello. Laúnica forma de probar dicho cumplimiento era (y sigue siendo) la presentación de todos los documentos "probatorios" de ese efecto. Sin este debido acompañamiento un libro no podía ser presentado en sociedad. En cierto modo el documento nossirve de forma más instrumental que el libro, es decir está elaborado bajo cierta intención, porlo que su vida es efímera en este contexto. El otro, el testimonial y jurídico, el más importante porque recubre al documento de inmortalidad y de gloria; nos referimos a su potencial histórico. 


\section{Investigacón Biblideedógia v. 13 No. 26 enem/ junio de 1999}

El documento habla, mientras que el libro susurra. No deberíamos darle mayor o menor importancia a ninguno de ellos por lo que se refiere al valor cultural que poseen. Pero sí debiéramos hacer hincapié en los elementos singulares de cada uno en esta valoración. El problema surge en el momento en que fijamos nuestra atención en los conjuntos, tanto de libros como de documentos. Es aquí donde debemos pre cisar el papel que desempeñan las instituciones encargadas de la tutela y el valor social que adquieren con esta custodia. Contexto que además explica y justifica, que el conjunto de estos materiales siempre esté considerado hacia el beneficio colectivo y por tanto el reconocimiento de la titularidad pública del dominio.

Si bien en el reconocimiento de su historia cada sociedad selecciona momentos importantes de grandes gestas del tipo que sean y dentro de éstas coloca a grandes hombres para remembrar, también es cierto que utiliza a los libros y documentos para contextualizar y justificar esta selección. D e esta forma el armazón que explica a la cultura misma, trasciende el tiempo y le otorga a ésta inmortalidad; porque sus contenidos se engendran en el deseo de perpetuar y de transmitir ${ }^{20}$ ya sea un saber, una experiencia o un pedazo de imaginación.

Y esta importancia cultural que reviste a ambos objetos (libros y documentos) está determinada por el valor que han alcanzado a lo largo de la historia. Este valor ha sido otorgado en parte por la representación y utilidad que tienen estos objetos para la sociedad, y en parte por su importancia fundamental como herramientas de comunicación. Hoy en día, ambos objetos en cierta medida han sido opacados por las virtudes inherentes a las herramientas de comunicación que el desarrollo tecnológico ha logrado a lo largo de este siglo. A síla realidad cotidiana, el acontecer actual, es decir "la noticia", se transmite por medios más eficaces: la radio, la televisión ylas redes de telecomunicación. Pero todo ello responde a la cultura de lo efímero y de la virtualidad que hoy nos inunda. Pero esta misma cotidianidad no puede ser explicada sin esos conjuntos bibliográficosy documentales que permanecen ala sombra.

Pero cuando hablamos de esta eficacia tecnológica no hacemos mención al efecto que se espera del establecimiento de una comunicación: un mensaje que se transmite y que se recibe, en donde las dos entidades juegan un papel fundamental del proceso. Nos referimos al valor que se otorga en las sociedades modernas a la información. Este término hace referencia a un conocimiento de lo que ocurre en espacios de tiempo reducidos. Estar informado significa por tanto saber qué acontece. Significa "estar al día" sobre lo que ocurre en el mundo y no sólo en el entorno propio sino tomando en cuenta otros acontecimientos sociales, políticos, culturales, económicos y, más aún, científicos y tecnológicos.

Estavida de las sociedades pendientes del respiro dela cotidianidad, se define y se establece con el establecimiento del tiempo moderno. Este concepto de modernidad constituye una ruptura categórica con un referente: el pasado. A través del encumbramiento del sentido de la antigüedad, es como la modernidad se instaura en la

20 José Antonio Pérez Rioja. Elogodd libro Madrid : CEG AL, 1995. p. 18 
sociedad. La sociedad como producto de la cultura occidental que nace, se reproduce y se difunde desde Europa. Es esta cultura la que funda la idea de modernidad a través del establecimiento de su concepción particular del paso del tiempo, y la que elabora una línea en temporal en la que se colocan cada una de las diferentes culturas; la suma de todas ellas será el concepto de universalidad.21 No obstante este concepto y estaforma de entender el mundo es algo que nos ha sido impuesto mediante el establecimiento de categorías absolutas cuya máxima expresión divide al mundo en sociedades modemas y sociedades primitivas.

El establecimiento de tales categorías se debe entre otras cosas, a razones históricas y a la supremacía que han alcanzado los valores occidentales y que se han introducido en otras culturas principalmente por medio dela colonización.22 Esta supremacía ha provocado que la valoración de los símbolos culturales se incline hacia algunos objetos pertenecientes a ciertas culturas privilegiadas en detrimento de otras, que en esta línea o bien no tienen lugar o no reconoce las mediciones de los otros. Esto se da porque la historia como ciencia, ha privilegiado ciertos acontecimientos en el tiempo en función de un cierto desarrollo de las sociedades, y en este sentido nos referimos al desarrollo del valor que se le otorga al bien cultural. A pesar de esto, "en todas partes se ha vuelto mayor la distancia entre ciencias históricasy el proceso público de tradición cultural. La fiabilidad del sabery el conflicto deinterpretaciones fomentan más la problematización de la conciencia histórica que la formación de identidad y la creación de sentido." 23

Si partimos de que el hombre (sin distinción alguna) es el productor de la historia y por tanto de la cultura, entonces tendremos que entender que no es posible otorgarle valor cultural a un objeto determinado sin más. Es necesario recordar que el bien cultural, el concepto de la Comisión Franceschini que hemos mencionado, está determinado por la referencia cultural que le permite ser identificado como tal. ${ }^{24} \mathrm{Y}$ he aquí la poción mágica, este reconocimiento necesariamente estará otorgado por la pertenencia cultural del objeto, pero ésta sólo pueden determinarla los agentes que lo producen y por tanto, lo valoran.

De aquí que en un grupo social ubicado en un mismo entorno geográfico, la discrepancia entre el valor cultural de tal o cual objeto estará determinada por la función simbólica involucrada. Y lo mismo ocurrirá entre culturas del todo diferentes. Todo sistema de significaciones culturales estará por tanto en completa relación con la identidad colectiva elaborada y reconocida por un devenir histórico determinado, lo que a su vez será condición necesaria para fundamentar todos y cada uno de los significados. Así, entre generaciones consecutivas, esos referentes constituyen una comprensión intersubjetiva.

21 Jean-Toussaint D esanti. "Sobre el tiempo, la historiay la historicidad”. En Historiaydivesidaddelas alturas Barcelona : Serbal, 1984. p. 190

22 Jacques Le G off. Pensarlahistoria Barcelona : Paidós, 1991. p. 134

23 Jürgen Habermas. Idantidadesnadionalesypostnadionales Madrid : Tecnos, 1989. p. 98

24 Juan Manuel Alegre Avila. Op at. p. 645 
Como sucede con los otros bienes culturales, el libro y el documento son apreciados desde diferentes perspectivas y por tanto su valor cultural es considerado de diferentes maneras. No obstante la importancia instrumental de estos bienes en la propia cultura ha traspasado fronteras, es decir su valoración es de carácter universal. A quí hacemos referencia más que al objeto físico ${ }^{25}$ al continente de aplicación: el contenido que le da personalidad propia einalienablea cadauno de ellos. Asílavaloración de una obra narrativa de Honoré de Balzac diferirá necesariamente de la que se haga sobre la obra de Charles D arwin. En el mismo sentido se distinguirán entre sí Las capitulaciones para la entrega de Granada y el acta de nacimiento de Jorge Luis Borges. La valoración del referente cultural estará determinada por los acontecimientos históricos que suceden en las culturas en las que se enmarcan. Lo que no elimina el valor cultural intrínseco de todos estos testimonios.

La cuestión es por qué les otorgamos a esos testimonios valor cultural fuera de sus condiciones materiales, históricas, e incluso geo gráficas y los denominamos bienes bibliográficos y documentales: porque el valor otorgado está mayoritariamente determinado por la función social que ambos objetos adquieren en el desarrollo de todas las culturas, e igualmente por su condición histórica. No son solamente productos de la cultura como cualquier o tro objeto, sino más aún, son y sobre todo germen de cultura. Y de aquí su importancia universal.

En este sentido, un monumento ${ }^{26}$ ocupando con su sola presencia un lugar en el espacio, no puede tener más que el valor rememorativo y el histórico. A mbos en re lación directa con la sociedad y su devenir en el tiempo. Pero si este mismo monumento es puesto como eslabón en una cadena de sucesos que se contextualizan y explican su origen en un continuo devenir histórico, adquieren una carga valorativa que le otorga el grupo social que realiza esta acción. Sólo entonces cobra un valor cultural recargado de significados. El monumento en cuestión puede o no gustarnos, pero de ninguna manera conflictúa nuestra existencia porque pertenece a nuestro entorno.

Una obra pictórica o arquitectónica sacada de su entorno natural, aquél en el que fue creado, pierde inevitablemente todo sentido temporal y referencial. No ocurre así con los libros y documentos, que se vuelven intemporales no sólo en el curso de la historia sino en función de cualquier otra distinción. Si bien es cierto que librosy documentos no pueden ser puestos en valor sin comprender las claves de su creación (tanto las particulares del sujeto creador como las del entorno del objeto creado), no requieren de estos elementos para obtener una personalidad

25 En tanto que no podemos hacer referencia a una delimitación específica de la materia de estos objetos en cuestión. Si bien el libro, puede identificarse de una cierta manera, dadas las condiciones planteadas en las definiciones puede a su vez ser documento. Sin embargo éste no puede ser libro, toda una paradoja.

26 Entendido éste como aquél objeto realizado por obra humana realizado para una finalidad específica y que contiene elementos presentes en la conciencia de las generaciones venideras. 
propia e intransferible. Porque las materias de su creación son el pensamiento humano y su vida social.

Un monumento, cualquiera que éste sea, nos remite única y exclusivamente a un tiempo y a un lugar de una cultura. Así, tanto la Pirámide de K eops como la Catedral de Notre-D ame son objetos que refieren a un momento histórico irrepetible que le otorga sentido eidentidad al propio objeto. Pero el objeto, incluso apreciado de esta forma, sólo nos puede dar una visión parcial de la historia y la cultura a la que pertenece. Mientras que los libros y documentos; el primero en solitario y el segundo necesariamente en familia; logran entregarno s alaluz del tiempo visiones más complejas de ese momento en que son creados. Ni siquiera un bien inmueble no podría trasmitir del todo su contenido cultural de no estar acompañado de los testimonios bibliográficos y documentales.

Así, la presencia de éstos es fundamental para conocer los otros bienes culturales, y por eso todo trabajo de restauración, rehabilitación o conservación de éstos, requiere necesariamente de una labor previa en donde el libro y el documento constituyen el fundamento de toda acción..$^{27}$ Es decir, se requiere de la reconstrucción histórica del objeto en cuestión para poder justificar cualquier política, y esto no puede hacerse sin la voz del libro y del documento.

Lo que les permite a estos objetos (los bibliográficos y los documentales) poseer esta visión integral, es algo que les proporciona el propio lenguaje. Es decir se vuelven de una u otra manera "conversaciones" con el complejo espíritu del hombre; con sus vertientes y sus formas. Un objeto artístico como una obra arquitectónica, refleja una utilidad que determina la sociedad que lo construye y que le confiere un sentido. Una iglesia, una mezquita, una muralla, una efigie ecuestre, etc. dicen de sí una función determinada e inalienable. En estesentido únicamenteel documento se acerca a ellos, mientras que el libro permanece y pertenece a otro contexto.

D e la misma manera el documento es creado con una intención y refleja una función específica. No es de ninguna manera un objeto atemporal y subjetivo, al contrario es totalmente objetivo porque emana en su contenido la forma en que se expresan la sociedad y sus normas. Manifiesta una intención nada fortuita y su existencia explica y reconoce una forma de entender esa actualidad en la que nace. Por ello el documento es también un monumento, ${ }^{28}$ en tanto que su presencia significa de antemano porque así fue creado." En contra de lo que a veces suelen imaginarse los principiantes, no surgen los documentos, aquí y allá, por el sólo efecto de no se sabe qué misterioso decreto de los dioses. Su presencia o su ausencia, en tales o cuales archivos, en una u otra biblioteca, en el suelo, depende de causas humanas que no escapan al análisis, y los problemas que plantea su transmisión, lejos de tener únicamente el mero alcance de ejercicios técnicos, rozan lo más íntimo de la vida del

27 Crr. Carlos Flores Marini. Retauradóndedudades México : FCE, 1976

28 Jacques Le Goff. Op at. p. 11 


\section{0}

pasado, porque lo que se encuentra así puesto en juego es nada menos que el paso del recuerdo a través de las generaciones." 29

Una cultura manifiesta su grandeza en el monumento, plasma en éste el interés del grupo humano como comunidad. D eja indicios en el camino de su paso por el tiempo y por tanto de su existir. Un monumento no se encuentra aislado, está y pertenece a un lugar que reflejala viday el sentir de la sociedad que lo erige; a través de él se identifica y adquiere conciencia tanto social como histórica. El monumento crea raíces que fortalecen el sentido del grupo social, de formatal que incluso en ausencia existe. Aunque el objeto material desaparezca, el sentido y la identidad cultural que le fueron otorgados permanece de forma tal que es posible encontrarlo y recrearlo en otro tiempo e incluso en otro lugar.

El objeto cultural puede ser valorado por la sociedad que le es contemporánea pese a que éste pueda desaparecer definitivamente. Porque es tal su fortaleza por lo que toca a la identidad del grupo como ente social, que permanece majestuoso e inalterable pese a su destrucción. Evidentemente para que esto sea así, es necesario que el colectivo social le haya otorgado ese valor cultural de identidad y de historia, de forma tal que se convierta en sinónimo referente de esa sociedad.

Porque para que ese monumento exista, el sentimiento queidentifica al colectivo humano debe haberse constituido y fortalecido de forma tal que lo más natural sea otorgarle al objeto todo ese cúmulo de significados. Podríamos ejemplificar esta afirmación recurriendo ala cultura egipcia. Para que fuese posiblela construcción de las pirámides de Gizeh, el grupo humano propio de esa cultura había tenido que desarrollar una sociedad y por tanto una forma especial de vivir en su entorno. Con ello tendría que haberle dado, por razones de conciencia histórica y social, un lugar preponderante a la figura del faraón, y por tanto, la construcción de la pirámide tenía ya en sí misma en el momento justo en que las piedras se unen para tomar esa forma singular, un valor cultural otorgado. Lo que significa que para el pueblo egipcio, la pirámide contenía una carga cultural. Es decir el revestimiento de símbolos permitía la creación de una pirámide y no de otra cosa.

El objeto requiere que le otorguemos un valor cultural para transfomarse en monumento. En el lapso en que fue creado respondía a una utilidad práctica necesariamente condicionada por su entorno. Está ahí y permanece, pero no únicamente como objeto material sino que retiene toda la carga cultural con que fue revestido inicialmente. A esa carga se le añade, por así decirlo, el peso de la historia. El objeto no se transforma, ni siquiera se modifica su valor cultural sino que se trasmite y se hace comprensible. Portodo ello es porlo que podemos hoy en día valorarlo en función de un tiempo y un lugar.

Pensemos un momento en Stonehenge, podemos suponer que en su tiempo y lugar debió haber sido un sitio quetuvo unafinalidad específica. Lamentablemente no

29 Marc Bloch. Apdogjeparl histaireaumátier d’histaien París : Colin, 1949. CitadoparJacquesLeGoff. “Doumento Monumento". EnIRARGI. No 2 (1989). p 120 
disponemos de "material" suficiente para comprender el verdadero valor cultural quetuvo antes y por tanto tendría hoy para nosotros. No obstante podemosvalorarlo como monumento por este sentido implícito, principalmente por su valor histórico. Lo que nos dice que de la misma manera que los hombres viven sus circunstancias, las obras arquitectónicas responden a ellas: no son gratuitas y mucho menos han salido de un sombrero negro igual que el conejo blanco.

Este es el caso del monolito, el arco, la muralla, la ciudad, la catedral, y un sinnúmero de objetos de cultura que hemos heredado. D e una u otra manera representan verdaderos anclajes de la historia de la humanidad. Por eso aunque su forma no sea igual, el documento debe ser para nosotros un monumento. Y debe serlo porque es el que mejor explica su propio sentido, aunque no todos los documentos poseen esta carga cultural en el momento en que son creados. Pero si observamos al documento como algo tan singular como lo es cada ser humano que vive en este lío que llamamos historia ¿por qué no pensar que también posee esa intencionalidad característica de los monumentos?. Incluso la razón misma de su existencia está marcada por esa intencionalidad prácticay directa. Sin la intencionalidad, el documento no se hace presente.

Pero si esto es así ¿por qué el libro no puede aspirar a tener esta misma condición?. No la tiene instricusensuya que el libro no posee del todo determinada esa intencionalidad. D igamos que el objeto libro en estamismalínea de argumentación, es ambivalente. Una catedral no puede ser otra cosa que una catedral y representa culturalmente su sentido y por tanto cumple con su intencionalidad. Un libro puede 0 no tener una intencionalidad claray, más aún, en el momento justo desu creación no está revestido de una carga cultural específica y delimitada. La obra pictórica se acerca más en este sentido al libro: un cuadro de del Rey Felipe IV pintado por Velázquez, estánecesariamente marcado por una intencionalidad, cumple una función específica y tiene por tanto una carga cultural. Pero otro cuadro del mismo autor que haya "surgido" en un momento de su inspiración no tendrá esa intencionalidad y por tanto carecerá de dicha carga cultural. Su contenido de cultura puede serle otorgado en el curso del tiempo, en el momento en que la obra del autor cobre otro sentido.

Será únicamente después de valorarlos culturalmente cuando este tipo de objetos podrán ser considerados como portadores de carga cultural. Sin embargo habría que plantearse una pregunta: ipor qué sólo ciertos autores y por tanto sus obras son revestidas de cierta carga cultural? Consideremos que el espíritu creador del hombrele pertenece a cada individuo de la especie de forma inalienable. Pero el problema de la creación radica sin lugar a dudas en la posibilidad de liberar y ser consciente de ese espíritu que nos habita. Yo podría tener en mi cabeza imágenes realmente hermosas pero si no sé pintar, o en todo caso si no poseo cierta habilidad, o no me he educado para ello, ciertamente no podré pintar una obra que trascienda en el tiempo.

Esta es la cuestión fundamental, sólo ciertos individuos logran alcanzar la inmortalidad, pero no únicamente por sus propias características singulares sino también porque son productos de su historia cultural. Es decir para que cada sujeto u "hombre 


\section{InnestigacónBiblictedógica V. 13 No. 26 enero/ junio de 1999}

ilustre" de la humanidad pueda alcanzar este reconocimiento debe ser considerado como un hombre de su tiempo. Quizás sólo sea que esas personas logran liberar a ese espíritu creador y sean conscientes de ello, y que en cierto modo sean libres de sí mismos. Pero esas peculiaridades que la historia de los hombres posee de vez en cuando no son productos fortuitos de la cultura humana. No es posible entenderlos sin revisar cuidadosamente sus antecedentes. A hí encontraremos con toda certeza uno que otro hombre o mujer que ya no existe pero que ha contribuido con fortaleza a la creación de un espínitu absoluto.

Por ello no es de extrañarnos que la mayor parte de estos seres humanos ilustres de la humanidad, siempre hayan tenido a su lado a los libros, creado con ellos un diálogo continuo entre el pensamiento pasado, y conformado poco a poco el presente. E s esta compañía la que ha ido moldeando poco a poco la silueta del espíritu creativo. Revisando las lecturas que hizo este espíritu, es posible acercarnos al tipo depensamientos que contribuyeron a crear un objeto cultural determinado.

Pero no sólo acompañan a estos hombres las hojas encuadernadas con un portentoso contenido, están también, casi sin que se den cuenta de ello, los documentos. Sí, porque éstos pasan sigilosamente por la vida de los hombres respondiendo a aquellos pequeños matices que por ser tan naturalesnos pasan desapercibidos. Esos documentos responden ala vida social, política, cultural y económica del entorno en que habitan los hombres.

La trágica vida de Vincent Van G ogh, es un buen ejemplo. Este hombre buscaba un "algo" dentro de sí; buscaba el color y además hacerse pintor. Todos los días ha cía estudios y se dedicaba con fervor al trabajo. Para ello leía sobre cuestiones que le atraían, y en particular sobre la pintura: "Tu tienes mejor que yo la ocasión de oír hablar de libros de arte. Cuando encuentres obras buenas, como por ejemplo el libro de Fromentin sobre los pintores holandeses, si te acuerdas de alguna de ellas, no olvides que deseo vivamente que compres algunas, que no sean de éstas que tienen la costumbre de enviarme y siempre tratan de técnica. Tengo la intención de aprender seriamentela teoría; no considero en absoluto ésto como inútil, y creo quea menudo lo que uno siente o capta instintivamente, se vuelve claro y seguro cuando se está guiado en sus búsquedas por algunos textos que tengan un sentido real práctico." 30 Conocemos detalles de la vida de este pintor por las cartas queescribió a su hermano cotidianamente. En estos documentos no sólo podemos apreciar su relación con el mundo sino las particularidades de la vida de los lugares en que Van Gogh estuvo. Incluso nos enteramos de ciertos gastos, de sus relaciones humanas y de cómo poco a poco su enfermedad acabaría con él.

El nacimiento de Van Gogh, como el de cualquiera, da lugar a la existencia de un documento que lo prueba. Q uiénes fueron sus padres y las ocupaciones que tenían, el dónde y el cuando de su historia personal son cuestiones que certifica un documento. También fue a una escuela y de ello sabremos por un documento, e incluso

30 Vincet Van G ogh. Cartasa Thé Colombia : Labor, 1992. p. 122 
podríamos averiguar qué clase de aprovechamiento académico tenía. Pero en el tiempo en que él existía no se creó ninguna carga cultural ni sobre su obra ni sobre su persona. La carga cultural que evoca la mención de su nombre se dio después de su muerte. Muere en 1890 y dos años después se presenta su primera exposición; de ahí en adelante la historia nos es bien conocida.

No es exagerado subrayar el papel que desempeña el documento en la valoración cultural. Si visitamos hoy cualquier exposición dedicada a uno de esos espíritus creativos encontraremos una multitud de documentos paralelamente expuestos junto con los propios objetos del sujeto en cuestión. Forman parte de la vida del individuo, es gracias a estos testigos silenciosos como podemos volver a recrear un pasado; más aún, con base a ellos podemos volver a darle forma a un objeto o persona hace tiempo desaparecido. Tanto el libro como el documento son compañeros de ese momento histórico, único e irrepetible; es por medio de estos dos bienes culturales que esa huella en el tiempo cobra fuerza y obtiene una silueta de vida y percepción que trastoca nuestro presente. ${ }^{31}$

Ésta es una de las cualidades por las que libros y documentos pueden ser considerados como bienes bibliográficos y documentales y por tanto bienes culturales. Su presencia en la vida de un sujeto lo transforma necesariamente, porque no sólo recrean un pasado sino que más aún, cuestionan la vidaindividual del sujeto. Este tipo de vinculación difícilmente la posee un monumento.

D e esta formalos objetos culturales se complementan y se requieren para formar una visión completa de ese pasado referencial que nos permite entender la actualidad que vivimos. Nada en la historia es gratuito, los hombres y por tanto sus vidas son resultado de las circunstancias. ${ }^{32}$ Éstas, en tanto acontecimientos en el tiempo y el lugar que le toca vivir a un hombre determinado, serán las fuentes de las que beberá su creación y su formación como sujeto. Nuevamente nos encontramos hablando de ese pieque se asienta en lo acontecido, de forma tal que sin él seríamos un punto en la nada.

¿Por qué si no, coleccionamos libros y documentos? ¿Por qué si no, los países se enorgullecen de sus bienes bibliográficos y documentales? ¿Cuál otro sentido si no es el que contienen como elementos de cultura tanto libros y documentos, justifica el interés de preservar no sólo aquello que se produjo, sino también lo que se está produciendo?. Porque existe la necesidad humana de conservar la memoria colectiva que se encuentra en esos objetos, cuya diversidad y cantidad, permite pensar que no pueden ser considerados únicamente como bienes bibliográficos y documentales aquellos que tienen valor histórico. En este sentido el alcance de la protección y la tutela no puede sólo realizarse sobre testimonios del pasado sino

31 Pedro Salmerón Escobar. "Monumento y ciudad" En Patrimmioyơudad Córdoba : Instituto Andaluz del Patrimonio Histórico : Ayuntamiento de Priego de Córdoba : Junta de Andalucía, 1994. p. 22

32 José O rtega y Gasset. El sentimientoestéticodela vicha Madrid : Tecnos, 1995. p. 68 
que debe también incluir a los del presente. Porque se comprenden únicamente en su relación continua.

Si bien es cierto que no podemos negar la trascendental importancia que tienen en este fin de siglo las nuevas tecnologías, tampoco podemos negar que falta mucho tiempo y paciencia para trasladar toda esa carga cultural preexistente en libros y documentos, a otro tipo de soporte, sea éste cual sea en el próximo siglo. El problema delos tiempos modernos es que la trascendencia de los objetos va más allá de su propia existencia física, hacemos de ellos "los dioses del fin del milenio". Nos olvidamos gravemente de que somos historia y de que para estar justo donde estamos hemos progresado. Pero más allá de nosotros, si nos pensamos como ese envase que envejece y muere, hay algo adentro aún innombrable. Ese "algo" que aprende y sobrevive en estemundo y su naturaleza, como una pequeña partede todos sus procesos.

El nuevo orden del mundo busca categorías universales, como hace la ciencia, pero se olvida que este mundo está construido y vive de pequeñas particularidades: las de cada uno de los hombres que lo habitan y lo ponen en movimiento. Porque ocurre que en ese intento de saberlo todo nos hemos quedado desnudos y sin saber de nosotros mismos. El hombre no es únicamente una maquina que funciona maravillosamente, hay algo que tenemos todos pero que sólo pocos saben liberar: el espíritu. Pero entre las tantas cosas que el hombre hace en la búsqueda de sí mismo está el proceso de la escritura. "Los hombres escriben ficciones porque están encarnados, porque son imperfectos. D ios no escribe novelas." 33

G racias a ese maravilloso proceso y búsqueda que es la escritura, tenemos ahora millones de libros y documentos, todos igualmente singulares eincomparables unos entre otros. Son únicosy por eso son considerados bienes patrimoniales. Resulta curioso observar que cada vez que se hace mención a este tipo de bien patrimonial, sabemos y entendemos qué lo conforma, pero muy pocas veces recordamos que dentro de esos objetos hay algo inmaterial que no es asequible a nadie en particular: la creación humana. La carga cultural de cualquiera de estos bienes patrimoniales es un peso añadido que trasciende el continente del objeto, es una carga simbólica que referencia y valida más que cualquier otra cosa, una cierta sensibilidad que por su propia naturaleza es inmaterial. Nos preocupamos mucho del objeto, pero poco del contenido cultural que palpita dentro de él. ${ }^{34}$

Por ello decimos que el patrimonio documental está conformado de tales y cuales documentos, de la misma forma que la conformación del patrimonio bibliográfico está configurado por tales y cuales libros. Son un cúmulo de objetos a los que les da mos una cierta importancia, a los que les añadimos una carga cultural. Pero dicha carga no es conferida de igual forma a todos los objetos de cultura, ni de igual manera

33 Ernesto Sábato. "Prólogo". En Historia y diversidad de las culturas. Op at. p. 100

34 Jorge Benavides Solís. "El componente cultural en el origen, la evolución y el contenido de los conjuntos históricos". En Bdéín Infomativodd InstitutoAndaluz de PatrimmioHistónico Año 3, no. 10 (marzo 1994). p. 28 
es valorada por el colectivo social que realiza este efecto. Un bien cultural precisamente se distingue de los otros por ser un referente simbólico dentro de la cultura. D e otra manerano existiría una "selección natural" de los objetos, y no habría un espacio capaz de contenerlos.

Dicha selección la realiza la propia sociedad en la que se crean e incluso los propios creadores de dicha obra. No todo representa, como no todo lo que representa es. Compleja circunstancia que responde a la compleja maquinaria que es el hombre y su entorno. Si a ello sumamos esta nueva tendencia a una "universalidad" 35 que borra de cierta manera las delimitaciones del cuerpo de cada cultura, el problema de la supervivencia de ese organismo se agrava rápidamente. Hoy en día los lugares tienen una cierta semejanza y para descubrir las diferencias es necesario observar con más detalle cual si fuésemos naturalistas.

Ante la tendencia hacia la universalidad, se presenta sin embargo una resistencia que en ocasiones se manifiesta con violencia y que nos hace olvidar que somos diferentes. No obstante, somos humanos y sólo una más de las especies vivas del planeta. Tenemos que respetar las diferencias culturales, entendiendo que no son sinónimo de desigualdad. Los bienes de la cultura son un patrimonio de todos, porque somos historia y nuestras "diferencias" se han mezclado. Ciertas cosas permanecen y hay que cuidarlas y perpetuarlas, en tanto que son singulares y nos dan cuenta de una entre miles de todas las visiones de la realidad de los seres humanos: pero esto no es nuestro privilegio, es nuestro deber. Los bienes culturales no nos pertenecen, siempre son del futuro y de las generaciones venideras, esa es su naturaleza. Así como tampoco podemos transformar estas diferencias en elementos que crean conflictos, la compleja naturaleza humana ya se encargará de ello, pues " todo lo que tocan los seres humanos se convierte en una mezcla de bueno y malo." 36 D ebemos tener presente que con todos los bienes patrimoniales tangibles e intangibles que se pierden en cada conflicto armado, perdemos todos y cada uno de los hombres. No sólo ha habido terribles pérdidas humanas, peor aún, hemos dejado que se eliminara la esencia de ellos y nunca más habrá siluetas. Estas son las cosas que tenemos que empezar a evitary para ello es necesario comprender que al defender estos bienes de naturaleza cultural, las disciplinas que se ocupan de su estudio deben abrirse y relacionarse con otras para recuperar el sentido en nuevas definiciones. Finalmente la cultura y sus productos no son más que el resultado de complejas relaciones sociales que no pueden ser abordadas desde un solo punto de vista.

35 Entendemos por esto la acción de fusionar la propia forma de existencia, en razón de uniformar todas las demás formas en el reconocimiento de igualdad.

36 Jostein Gaarder. El mundodeSofía Barcelona : Círculo de Lectores, 1995. p. 518 